Peipei Wang, Lingling Zhao*

\title{
The Expression Change of GFAP and HMGB1 in Primary Cultured Astrocytes Exposed to Chlorpyrifos and Lipopolysaccharide
}

\begin{abstract}
Background and Objective: During the development of the nervous system, the impact of adverse environmental factors can cause serious consequences. Chemicals, toxic substances and infections are considered as common risk factors. In order to find the impact of environmental pollutants pesticides and infections on the nervous system, we attempt to investigate the effect of CPF (chlorpyrifos) and LPS (lipopolysaccharide) combined exposure on the release of GFAP/HMGB1 and activation in the primary cultured astrocyte of neonatal rat cerebral cortex. Methods: In this paper, trypsin digestion was used to isolate and purify astrocytes, and identify the cell based on immunofluorescence method; Astrocyte in experiment group was exposed to different concentrations of LPS and CPF. Astrocyte's activities were observed via CCK-8; Immunofluorescence and Western blot were used to measure the changes on the expression level of GFAP as well as HMGB1 in astrocyte. Results: Astrocyte's synaptic network was significantly increased at $24 \mathrm{~h}$ after being exposed to $25,50 \mu \mathrm{MCPF}$ combined with $1 \mu \mathrm{g} / \mathrm{mL}$ LPS; The expression level of glial fibrillary acidic protein (GFAP). High mobility group box 1(HMGB1) increased after astrocytes were exposed to $25,50 \mu \mathrm{M}$ CPF combined with $1 \mu \mathrm{g} / \mathrm{mL}$ LPS $(\mathrm{p}<0.05)$, The expression level of GFAP and HMGB1 decreased after being exposed to $100 \mu \mathrm{M}$ CPF combined with $1 \mu \mathrm{g} / \mathrm{mL}$ LPS $(\mathrm{p}<0.05) .4)$ Conclusion: 1 . CPF and LPS can not only cause astrocyte activation but also increase the expression of GFAP and HMGB1. 2. The effect of CPF combined with LPS is more obvious than CPF or LPS respectively on astrocytes, which indicates that they have a synergistic effect.
\end{abstract}

Keywords: chlorpyrifos, lipopolysaccharide, astrocytes, GFAP, HMGB1.

\section{Introduction}

For more than a century, many researchers have mainly focused on early life as a source of adult disorder. As suggested by recent studies, the complex interactions of genes and environment may be the cause of Neurodevelopmental Disorders [1]. Pesticides are common environmental chemicals, and people are usually exposed

\footnotetext{
${ }^{*}$ Corresponding author: Lingling Zhao, Pediatrics, The Third Xiangya Hospital of Central South University (CSU), Changsha, China, E-mail: Ilzhao2011@qq.com, fureya711@qq.com

Peipei Wang, Pediatrics, The Third Xiangya Hospital of Central South University (CSU), Changsha, China
} 
to low doses of pesticides over a long term period. However, there are no obvious symptoms of pesticide poisoning. With in-depth studies on the low doses of pesticides, their harmful effects are increasingly recognized by the public [2]. During the development process, infection is a common cause of illness. Although current studies focus on single factors, the growth process is often the combined action by the environment in a variety of factors [3]. Recently, double-hit models are used to study chronic/tonic pain [4] as well as stress dysregulation [5]. To analyze whether the interaction between the infection and pesticides will significantly affect the nervous system, we designed an in vitro study to investigate the double-hit of CPF and LPS, and selected astrocytes as targets, GFAP and HMGB1 as an index, so as to provide new ideas to combat Neurodevelopmental Disorders.

\section{Methods}

\subsection{Cell Culture}

Primary astrocyte cultures were prepared from the cerebral cortices of 1 to 3-dayold SD rats. Furthermore, isolated cells were maintained in DMEM/F12 (HyClone), supplemented with $10 \%$ of fetal bovine serum in an atmosphere of $5 \% \mathrm{CO}_{2} /$ humidified air $(95 \%)$ at $37^{\circ} \mathrm{C}$. After cells enrichment, Astrocyte was detached from the culture flask with $0.1 \%$ trypsin and $0.04 \%$ EDTA in Hank's balanced salt solution (SigmaAldrich). Notably, this was repeated three times. Astrocyte was plated onto poly-Llysine-coated glass coverslips, and supplemented with culture medium. Experiments were carried out 2-3 days after cell plating.

\subsection{Intervention Method}

The DMEM/F12 culture mediums with different chlorpyrifos and lipopolysaccharide concentrations were utilized respectively to cultivate the prepared astrocyte for 12/24/48 hours. After that, PBS washed cells can be applied for the next processing.

\subsection{Immunofluorescence}

Isolated cells were characterized by the primary antibody against GFAP as well as its corresponding secondary fluorescent antibody, and by the blue fluorescent nuclear stain DAPI. 


\subsection{Western Blot}

The whole astrocytes were prepared as described above. Meanwhile, the protein concentration was determined using a protein assay kit, and the separation method is SDS-PAGE. Then, the gel was electroblotted onto a nitrocellulose membrane. The membranes were probed with anti-GFAP (1:400 dilution), anti-HMGB1 (1:1000 dilution). Furthermore, filters were washed and incubated for $2 \mathrm{~h}$ at the room temperature.

\section{Results}

\subsection{CPF and LPS Activated Astrocytes}

Primary cultured neonatal rat's brain astrocytes exposed to LPS $(1 \mu \mathrm{g} / \mathrm{mL})$ combined with chlorpyrifos $(50 \mu \mathrm{M})$ established cell model, DMSO group, PBS group, chlorpyrifos $(50 \mu \mathrm{M})$ group, lipopolysaccharide $(1 \mu \mathrm{g} / \mathrm{mL})$ group in the control group. Moreover, GFAP expression was observed by immunofluorescence after $24 \mathrm{~h}$. CyTM3- red: GFAP expression; DAPI- blue: nuclei. A: After the DMSO treated $24 \mathrm{~h}$; B: PBS treated $24 \mathrm{~h}$; C: CPF $(50 \mu \mathrm{M})$ treated $24 \mathrm{~h}$; D: LPS $(1 \mu \mathrm{g} / \mathrm{mL})$ treated $24 \mathrm{~h}$; E: LPS $(1 \mu \mathrm{g} / \mathrm{mL})+$ CPF $(50 \mu \mathrm{M})$ treated $24 \mathrm{~h}$.

In this study, GFAP antibody was labeled with a red fluorescent dye CyTM3 that astrocytes cells were red. As shown by the results, both the star-shaped glial cells and synaptic contact increased after the exposure to LPS combined with low-dose chlorpyrifos for $24 \mathrm{~h}$. These findings suggested that astrocytes were active (Figure 1).
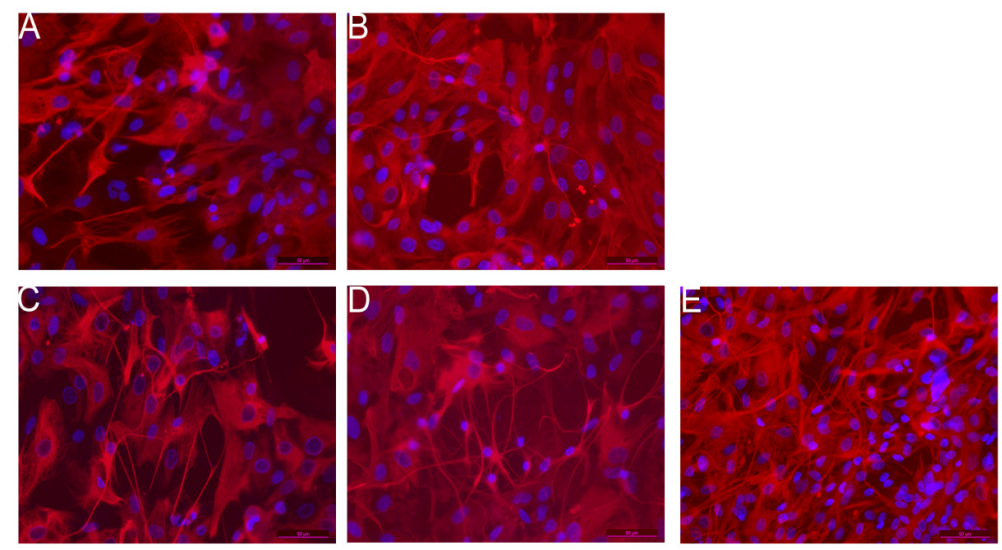

Figure 1: Expression of GFAP by astrocytes exposed to chlorpyrifos and lipopolysaccharide (immunofluorescence, $\times$ 400). 


\subsection{CPF and LPS Influences the Expression of GFAP}

Primary cultured neonatal rat cortical astrocytes were exposed to chlorpyrifos ( $25 \mu \mathrm{M}$, $50 \mu \mathrm{M}, 100 \mu \mathrm{M})$ and LPS (1 ug/mL); chlorpyrifos ( $25 \mu \mathrm{M}, 50 \mu \mathrm{M}, 100 \mu \mathrm{M})$ group, LPS (1 ug/mL) group, PBS (0.1\%) groups, and DMSO (0.1\%) in the control group. GFAP expression was observed by western blot after 12, 24, $48 \mathrm{~h}$. A figure shown GFAP protein expression to detect changes at different time points, $\beta$-actin as internal control. $\mathrm{B}$ is a chart GFAP protein expression. ${ }^{\star}$ : $\mathrm{P}<0.05$; $\mathrm{x}$, group compared with the same time PBS and DMSO; a: compared with the same time CPF, b: compared with the same time LPS, compared with different time points, c: compare to $12 \mathrm{~h}$, d: compare to $24 \mathrm{~h}$, e: compare to $48 \mathrm{~h}$.

The expression of GFAP was examined after astrocytes were simultaneously exposed to low-dose CPF and LPS via Western blot. As displayed by the results, the expression of GFAP in astrocytes exposure to chlorpyrifos $(25,50 \mu \mathrm{M})$ and LPS was gradually increased than the control group $(\mathrm{p}<0.05)$, while chlorpyrifos $(100 \mu \mathrm{M})$ and LPS group was decreased ( $\mathrm{p}<0.05)$ (Fig. 2).

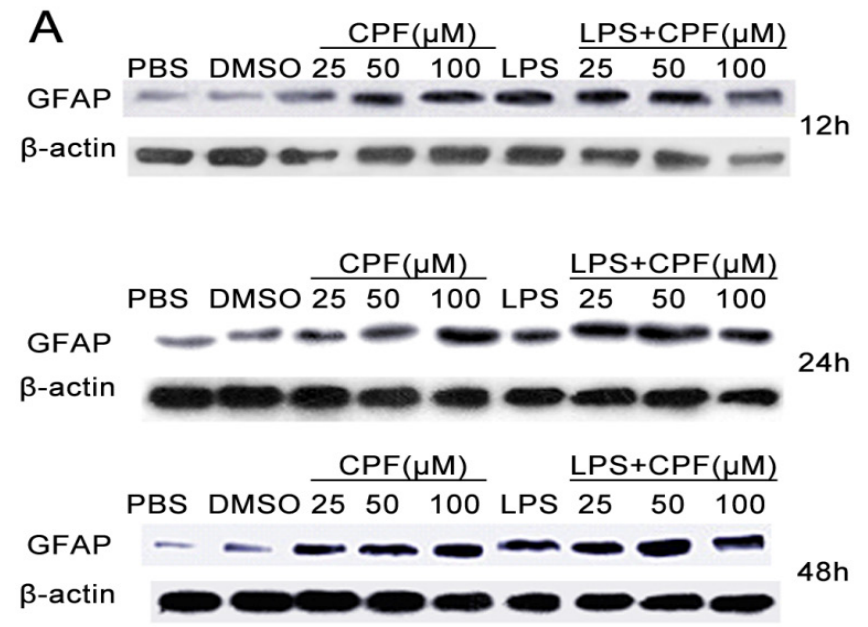

B

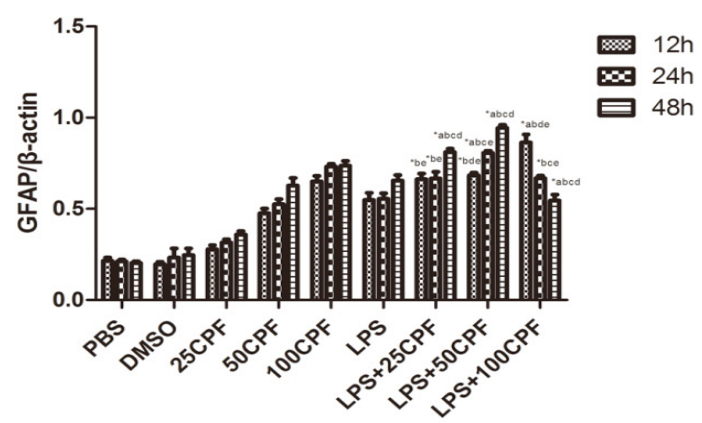

Figure 2: Expression of GFAP by astrocytes exposed to chlorpyrifos and lipopolysaccharide 


\subsection{CPF and LPS influences the expression of HMGB1}

Primary cultured neonatal rat cortical astrocytes were exposed to chlorpyrifos ( $25 \mu \mathrm{M}$, $50 \mu \mathrm{M}, 100 \mu \mathrm{M})$ and LPS (1 ug/mL); chlorpyrifos $(25 \mu \mathrm{M}, 50 \mu \mathrm{M}, 100 \mu \mathrm{M})$ group, LPS $(1 \mathrm{ug} / \mathrm{mL})$ group, PBS (0.1\%) groups, and DMSO (0.1\%) in the control group. HMGB1 expression was observed by western blot after 12, 24, $48 \mathrm{~h}$. A figure shown GFAP protein expression to detect changes at different time points, $\beta$-actin as internal control. $\mathrm{B}$ is a chart GFAP protein expression. *: $\mathrm{P}<0.05$; $\mathrm{x}$, group compared with the same time PBS and DMSO; a: compared with the same time CPF, b: compared with the same time LPS, compared with different time points: c: compare to $12 \mathrm{~h}$, d: compare to $24 \mathrm{~h}$, e: compare to $48 \mathrm{~h}$.

The expression of HMGB1 was examined after astrocytes were simultaneously exposed to low-dose CPF and LPS via Western blot. As shown by the results, the expression of GFAP in astrocytes exposure to chlorpyrifos $(50 \mu \mathrm{M})$ and LPS was gradually increased than the control group $(\mathrm{p}<0.05)$, while chlorpyrifos $(100 \mu \mathrm{M})$ and LPS group was decreased $(\mathrm{p}<0.05)$ (Fig. 3).
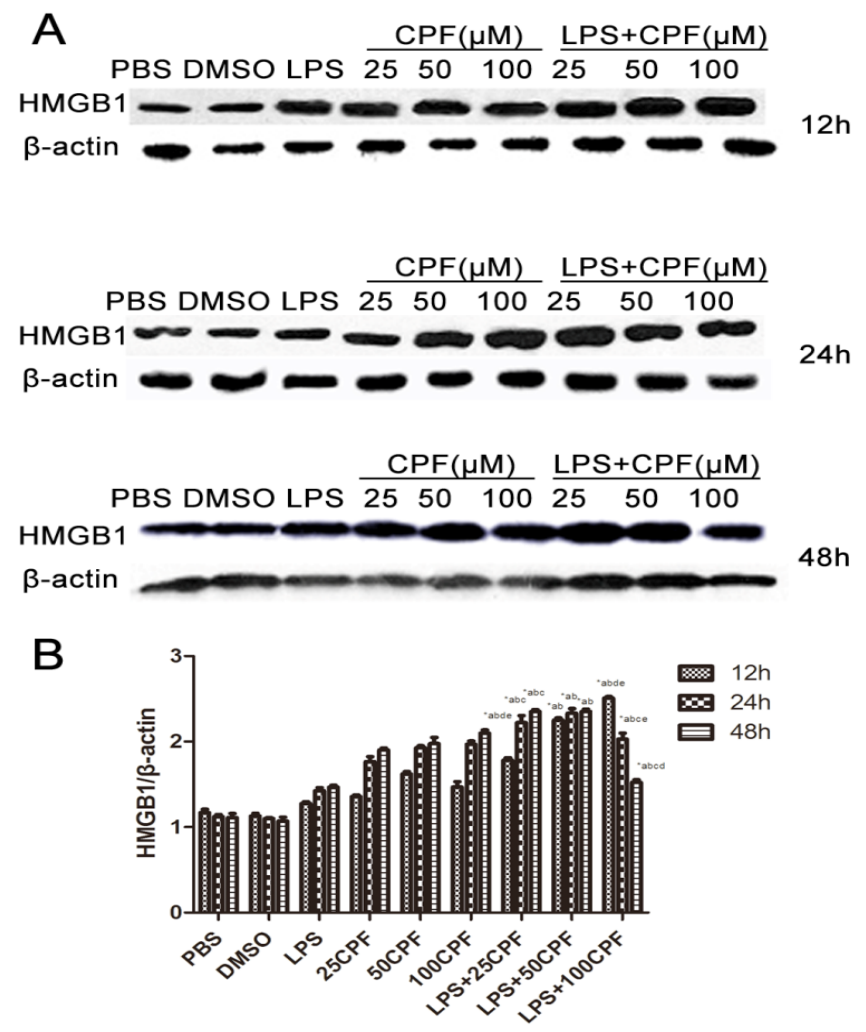

Figure 3: Expression of HMGB1 by astrocytes exposed to chlorpyrifos and lipopolysaccharide 


\section{Discussion}

This experiment attempts to study a double-hit effect on the nervous system from an environmental chemical pollutant pesticides and infections, so as to explore the interaction between these two effects. Additionally, the primary cultured astrocytes were exposed to low-dose CPF and LPS in vitro. As shown by the results, low doses of CPF and LPS change its expression of GFAP and HMGB1. Moreover, the effect of their interaction is more obvious than their effect alone.

Generally speaking, the environmental pollution is considered as a risk factor for Neurodevelopmental Disorders [6]. According to the analysis of some scholars [7], the relationship between the environmental pollution and neurodevelopmental disorders and environmental attributable fraction (EAF) is 10\%. People are often passively exposed to pesticides over a long period of time, and its health effects have attracted people's attention. Does the pesticide applied conform to "pesticide residue standards"? Is it really safe? A few studies have proposed that the "safe level" of toxic chemical residues is associated with neurodevelopmental disorders [8-9]. Organochlorine pesticides are persistent, and bioaccumulative environmental contaminants have potential neurotoxic effects. Moreover, the prenatal exposure to organochlorines is associated with impairment of neuropsychological development [10]. There is a growing emphasis on the impact of chlorpyrifos on children's neurodevelopment. As shown by animal or social investigation, the exposure to chlorpyrifos is closely related to children' nervous system damage [11], and it may cause neurodevelopmental impairment [10], mental retardation [12-14], ADHD [15], memory loss [16], anxiety and autism [17].

Previous studies from our group suggested that the sub-toxic doses of chlorpyrifos will induce brain inflammation [2], by HMGB1/TLR/NF-kB pathway in the brain amygdala regulated pro-inflammatory cytokines. Additionally, the HMGB1 could be the target of intervening and treating chlorpyrifos neurotoxicity [18].

Infections are a common reason attributed to sick children. As indicated by many studies, the intrauterine infection caused by fetal inflammatory response syndrome is the most common brain injury in preterm. The reason may be related to children's cognitive developmental disorders [19-21]. Inflammation is not only a potent inhibitor of neural development but also a high risk factor of cognitive impairment and depression [22-23]. A prospective study showed that $65 \%$ of very low birth weight children (gather of more Medical Center, a total of 6093 cases of newborns) had at least 1 infection. Compared with normal children, early childhood infections would significantly increase the risk of Neurodevelopmental Disorders [24].

The proliferation and differentiation of glial cells are later than neuron [25] because glial cells may be often affected by external factors during the maturity period. Many studies have suggested that the main target of chlorpyrifos is astrocytes [26-28]. Astrocytes refer to the important glial cells. Its features include the transfer guide neurons, glutamate and $y$ - amino butyric acid (GABA) metabolism, cell buffer 
of changes to the external environment, contact neurons through synapses. Also, it can accept a variety of materials through various receptor regulations, which results in nutritional factor [29]. GFAP is astrocytes' activation marker [30]. Moreover, a hallmark of gliotic reaction is the up-regulation of the astrocytic biomarker GFAP, which often precedes the anatomically apparent damage in the brain [31]. As shown by the experiments, CPF combined with LPS can give rise to the increase of astrocytes' expression of GFAP, suggesting that both CPF and LPS can cause astrocyte activation and nervous system damage. It is more obvious than each intervention alone, indicating that the two works together can jointly increase the activation of astrocytes and damage.

The important activity and function of HMGB1 lies in that it can be combined with various proteins. Also, it can mediate inflammation and gene transcription [32]. HMGB1 induces an inflammatory response via RAGE (receptor for advanced glycation end-products, RAGE) by glycosylation and Toll-like receptor family (toll-like family of receptors, TLRs) [33-34]. In this study, it was shown that CPF and LPS can cause the increase of HMGB1 expression in astrocytes, suggesting that CPF and LPS will work together to increase the nervous system injury. If the expression and secretion of HMGB1 is inhibited, can it alleviate the toxic effects of CPF and LPS on the nervous system? Can HMGB1 act as a common therapeutic target on these two kinds of injuries? This still needs further research.

\section{Conclusions}

From this study, it can be found that CPF and LPS will not only cause astrocyte activation but also increase the expression of GFAP and HMGB1. Additionally, the effect of CPF combined with LPS is more obvious than CPF or LPS respectively on astrocytes, suggesting that they pose a synergistic effect.

\section{References}

[1] Bale T L, Baram T Z, Brown A S, et al. Early Life Programming and Neurodevelopmental Disorders[J]. Biological Psychiatry, 2010, 68(4): 314-9.

[2] Zhang J, Dai H, Deng Y, et al. Neonatal chlorpyrifos exposure induces loss of dopaminergic neurons in young adult rats[]]. Toxicology, 2015, 336: 17-25.

[3] Gentile I, Altieri L, Lintas C, et al. Urinary polyomavirus infections in neurodevelopmental disorders[J]. Open Journal of Psychiatry, 2013, 03(2A): 18-25.

[4] Parent A J, Tétreault $P$, Roux $M$, et al. Descending nociceptive inhibition is modulated in a time-dependent manner in a double-hit model of chronic/tonic pain[J]. Neuroscience, 2016, 315(3): 70-78.

[5] Cotella E M, Durando P E, Suárez M M. A double-hit model of stress dysregulation in rats: implications for limbic corticosteroid receptors and anxious behavior under amitriptyline treatment.[J]. Stress-the International Journal on the Biology of Stress, 2014, 17(3): 235-46. 
[6] Kishi R, Kobayashi S, Ikeno T, et al.Ten years of progress in the Hokkaido birth cohort study on environment and children's health: cohort profile--updated 2013. Environ Health Prev Med[J]. 2013, 18(6): 429-50.

[7] Philip J. Landrigan, Clyde B. Schechter, Jeffrey M. Lipton, et al. Environmental Pollutants and Disease in American Children: Estimates ofMorbidity, Mortality, and Costs for Lead Poisoning, Asthma, Cancer, andDevelopmental Disabilities[J].Environmental Health Perspectives.2002, 110(7): 721-728.

[8] Lisa M Chiodo, Sandra W Jacobson,Joseph L Jacobson. Neurodevelopmental effects of postnatal lead exposure at very low levels[J]. Neurotoxicology and Teratology. 2004, 26(3): 359-371.

[9] Mendola P, Selevan S G, Gutter S, et al. Environmental factors associated with a spectrum of neurodevelopmental deficits[J]. Mental retardation and developmental disabilities research reviews, 2002, 8(3): 188-197.

[10] Seyed Soheil Saeedi Saravi, Ahmad Reza Dehpour.Potential role of organochlorine pesticides in the pathogenesis ofneurodevelopmental, neurodegenerative, and neurobehavioraldisorders: A review[J]. Life Sciences.2016(145): 255-264.

[11] Rauh VA, Perera FP, Horton MK, et al. Brain anomalies in children exposed prenatally to a common organophosphate pesticide[J]. Proc Natl Acad Sci U S A, 2012, 109(20): 7871-7876.

[12] Bouchard MF, Chevrier J, Harley KG, et al. Prenatal exposure to organophosphate pesticides and IQ in 7-year-old children[J]. Environ Health Perspect, 2011, 119(8): 1189-1195.

[13] Rauh V, Arunajadai S, Horton M, et al. Seven-year neurodevelopmental scores and prenatal exposure to chlorpyrifos, a common agricultural pesticide[J]. Environ Health Perspect, 2011, 119(8): 1196-1201.

[14] Engel SM, Wetmur J, Chen J, et al. Prenatal exposure to organophosphates, paraoxonase 1, and cognitive development in childhood[J]. Environ Health Perspect, 2011, 119(8): 1182-1188.

[15] Fortenberry G Z, Meeker J D, Sanchez B N, et al. Urinary 3,5,6-trichloro-2- pyridinol (TCPY) in pregnant women from Mexico City: distribution, temporal variability, and relationship with child attention and hyperactivity[J]. Int J Hyg Environ Health, 2014, 217(2-3): 405-412.

[16] Horton M K, Kahn L G, Perera F, et al. Does the home environment and the sex of the child modify the adverse effects of prenatal exposure to chlorpyrifos on child working memory?[J]. Neurotoxicol Teratol, 2012, 34(5): 534-541.

[17] Aldridge JE, Levin ED, Seidler FJ, et al. Developmental exposure of rats to chlorpyrifos leads to behavioral alterations in adulthood, involving serotonergic mechanisms and resembling animal models of depression[J]. Environ Health Perspect, 2005, 113(5): 527-531.

[18] Tian J, Dai H, Deng Y, et al. The effect of HMGB1 on sub-toxic chlorpyrifos exposure-induced neuroinflammation in amygdala of neonatal rats[J]. Toxicology, 2015, 338: 95-103.

[19] Volpe Joseph J. Neonatal encephalopathy: an inadequate term for hypoxic-ischemic encephalopathy[J]. Ann Neurol. 2012, 72(2): 156-166.

[20] Adib-Conquy M, Cavaillon JM, Jacqmin S, et al. CD24-triggered caspase-dependent apoptosis via mitochondrialmembrane depolarization and reactive oxygen species pro-duction of human neutrophils is impaired in sepsis[J]. J Immunol, 2014, 192(5): 2449-2459.

[21] Kaur C, Rathnasamy G, Ling E A. Roles of activated microglia in hypoxia induced neuroinflammation in the developing brain and the retina[J]. Journal of Neuroimmune Pharmacology, 2013, 8(1): 66-78.

[22] Zonis S, Ljubimov V A, Mahgerefteh M, et al. p21Cip restrains hippocampal neurogenesis and protects neuronal progenitors from apoptosis during acute systemic inflammation[J]. Hippocampus, 2013, 23(12): 1383-1394.

[23] Eisch A J, Petrik D. Depression and hippocampal neurogenesis: a road to remission?[J]. Science (New York, NY), 2012, 338(6103): 72. 
[24] Stoll B J, Hansen N I, Adams-Chapman I, et al. Neurodevelopmental and growth impairment among extremely low-birth-weight infants with neonatal infection[J]. Jama, 2004, 292(19): 2357-2365.

[25] C. Guerri, J. Renau-Piqueras, Alcohol, astroglia, and brain development[J]. Mol. Neurobiol. 1997.15: 65-81.

[26] Krishnan K, Mitra N K, Yee L S, et al. A comparison of neurotoxicity in cerebellum produced by dermal application of chlorpyrifos in young and adult mice[J]. Journal of Neural Transmission, 2012, 119(3): 345-352.

[27] Salyha Y T. Chlorpyrifos leads to oxidative stress-induced death of hippocampal cells in vitro[J]. Neurophysiology, 2013, 45(3): 193-199.

[28] Lee J E, Lim M S, Park J H, et al. Nuclear NF-кB contributes to chlorpyrifos-induced apoptosis through p53 signaling in human neural precursor cells[J]. Neurotoxicology, 2014, 42: 58-70.

[29] Mercier F, Kitasako J T, Hatton G I. Anatomy of the brain neurogenic zones revisited: fractones and the fibroblast/macrophage network[J]. Journal of Comparative Neurology, 2002, 451(2): 170-188.

[30] Orre M, Kamphuis W, Osborn L M, et al. Isolation of glia from Alzheimer's mice reveals inflammation and dysfunction[J]. Neurobiology of aging, 2014, 35(12): 2746-2760.

[31] Ho G, Zhang C, Zhuo L. Non-invasive fluorescent imaging of gliosis in transgenic mice for profiling developmental neurotoxicity[J]. Toxicology and applied pharmacology, 2007, 221(1): 76-85.

[32] Yang H, Tracey K J. Targeting HMGB1 in inflammation[J]. Biochimica et Biophysica Acta (BBA)-Gene Regulatory Mechanisms, 2010, 1799(1): 149-156.

[33] Huebener P, Pradere J P, Hernandez C, et al. The HMGB1/RAGE axis triggers neutrophil-mediated injury amplification following necrosis[J]. The Journal of clinical investigation, 2015, 125(2): 539.

[34] Wang X, Sun R, Wei H, et al. High-mobility group box 1 (HMGB1)-Toll-like receptor (TLR) 4-interleukin (IL)-23-IL-17A axis in drug-induced damage-associated lethal hepatitis: Interaction of $ү \delta$ T cells with macrophages[J]. Hepatology, 2013, 57(1): 373-384. 